\title{
PROOF THEORY AND MEANING: ON THE CONTEXT OF DEDUCIBILITY
}

\author{
GREG RESTALL
}

\begin{abstract}
I examine Belnap's two criteria of existence and uniqueness for evaluating putative definitions of logical concepts in inference rules, by determining how they apply in four different examples: conjunction, the universal quantifier, the indefinite choice $\bullet$ perator and the necessity in the modal logic S5. This illustrates the ways that definitions may be evaluated relative to a background theory of consequence, and the ways that different accounts of consequence provide us with different resources for making definitions.
\end{abstract}

The idea is compelling to many of us: The concept of conjunction is given fully in its rules of inference. Everything we need to know about the behaviour of "and" in the logicians' sense than is given by rules like these.

$$
\frac{p}{p \wedge q}[\wedge I] \quad \frac{p \wedge q}{p}\left[\wedge E_{1}\right] \quad \frac{p \wedge q}{q}\left[\wedge E_{2}\right]
$$

The virtues of this view are simple: When we learn inference rules, we learn how to use the connectives in deductive reasoning. One does not need to be a disciple of Wittgenstein to think that there is a connection between meaning and use, and in inference rules like these the connection with use is present to hand. The $[\wedge I]$ rule tells us how to get a conjunction, and the $[\wedge E]$ rules tell us what we can do with a conjunction when we have it. Inference rules speak to the matter of the use of the concept of conjunction without positing some "semantic value" to be the referent (or extension or whatever) of the concept.

Of course, there are very many issues with this way of looking at things. For example, while determinate and precise inference rules are available for many logical particles such as connectives, operators, quantifers and the like, it is harder to see how one might present other meanings in the same fashion ${ }^{1}$. The issue I wish to focus on in this paper is not the general problem of giving an account of meaning in terms of rules of inference. Instead, I wish to concentrate

This paper is a draft, and comments from readers are very welcome. Please check the webpage http://consequently.org/writing/ptm-context for the latest version of the paper, to post comments and to read comments left by others.

${ }^{1}$ This does not mean that there aren't those who try. Inferentialists, such as Robert Brandom [5, 6] are committed to giving an account of meaning in terms of inferential propriety.

Logic Colloquium '07

Edited by Françoise Delon, Ulrich Kohlenbach, Penelope Maddy, and Frank Stephan

Lecture Notes in Logic, 35

(C) 2010, Asseciation For SYMBOLIC Logic 
on the more local issue of the propriety of defining logical concepts in this way. Can one present inference rules and take them to define a connective, an operator or a quantifier? Is such a definition always legitimate, or are potential definitions beholden to some higher court of appeal? If logical concepts are freely definable, then what are the boundaries of such freedom? More interestingly still, what are we doing when we define a logical concept in this way?

The most striking formulation of this issue is due to Arthur Prior, in his influential paper "The Runabout Inference Ticket" [19]. He asks us to consider the binary connective "tonk" given by these two inference rules.

$$
\frac{p}{p \text { tonk } q}[\text { tonk }] \quad \frac{p \text { tonk } q}{q} \text { [tonkE] }
$$

If we can freely define connectives with inference rules, we have defined something. However, using the concept tonk which we have apparently introduced in a definition, we may infer any conclusion from any premise:

$$
\frac{p}{\frac{p \text { tonk } q}{q}[\operatorname{tonk}]}[\text { tonk } E]
$$

This is too much. Something has gone wrong in our definition. But what?

Responses to Prior's paper vary. One tradition is represented in J. T. Stevenson's "Roundabout the Runabout Inference-Ticket" [23]. Stevenson argues that tonk is unacceptable because the rules impose different truth-table conditions on tonk. According to [tonkI], $p$ tonk $q$ must be true when $p$ is true. According to [tonk $E$ ], when $q$ is false, $p$ tonk $q$ must be false. Since no connective can meet both of those conditions, there is no connective like tonk. There is more than one way of understanding Stevenson's criteria. The straightforward way is to take the possibility of a truth table definition for a connective like tonk as a criterion for its definability. This is to give the game ( defining the connective proof-theoretically) away. Truth tables are where the real action is.

This is not the approach I wish to consider. Instead, I wish to look again at the other kind of response to Prior's paper, that given by Nuel Belnap in his "Tonk, Plonk and Plink" [3]. For Belnap, criteria governing the acceptability of a definition can be given in proof theoretical terms. What makes tonk unacceptable is not some categorical feature of tonk, but in the interaction of tonk with the inference rules we already endorse.

It seems to me that the key to a solution lies in observing that even on the synthetic view, we are not defining our connectives $a b$ initio, but rather in terms of an antecedently given context of deducibility, concerning which we have some definite notions. By that I mean that before arriving at the problem of characterizing 
connectives, we have already made some assumptions about the nature of deducibility [3, page 131].

The point is that rules for tonk are given relative to a background set of commitments concerning inference. (Which may involve, for example, the commitment that the argument from $p$ to $q$ is invalid.) If the addition of the rules for tonk change our commitments concerning inference (we now have a proof from $p$ to $q$ ), then we are forced with a choice. Either reject those rules, or modify our original assumptions. Both are options. If we endorse the antecedent theory - or what Belnap calls our antecedently given context of deducibility, then this gives us a standard by which we can judge putative definitions.

We may now state the demand for the consistency of the definition of the new connective, plonk, as follows: the extension must be conservative; i.e., although the extension may well have new deducibility-statements, these new statements will all involve plonk. The extension will not have any new deducibility-statements which do not involve plonk itself. It will not lead to any deducibilitystatement not containing plonk, unless that statement is already provable in the absence of the plonk-axioms and plonk-rules. The justification for unpacking the demand for consistency in terms of conservativeness is precisely our antecedent assumption that we already had all the universally valid deducibility-statements not involving any special connectives [3, page 132].

On these grounds, if the background account of deducibility included the commitment that some $p$ did not entail some $q$, then, relative to that background, tonk fails the demand of consistency. This is one of the tests Belnap considers in the paper. In the case of a natural deduction proof theory or a sequent calculus, we can demonstrate that this criterion is met by means of a normalisation proof or a cut elimination argument, which usually has as a consequence a subformula property. These proof-theoretical results show that if one has a proof for some argument (or a derivable sequent) then we can find a special normal proof for that argument (or a cut-free derivation of that sequent) in which all formulas used in that proof are sub-formulas of the formulas in the premises or conclusion (or are subformulas of the formulas in the target sequent). This means that if the proof (or sequent) does not use our newly defined connective, then it has a normal proof (or cut-free derivation) not using the rules for our newly defined connective, for the rules for this connective must involve it somewhere, and the normal proof (cut-free derivation) does not feature this connective at all. It follows that if the argument is proved (sequent is derived) in the system using the new concept, then it can still be proved (derived) in the system before its introduction. 
Belnap considered another criterion to add to the criterion of conservative extension.

The mathematical analogy leads us to ask if we ought not also to add uniqueness as a requirement for connectives introduced by definitions in terms of deducibility (although clearly this requirement is not as essential as the first, or at least not in the same way). Suppose, for example, that I propose to define a connective plonk by specifying that $B \vdash A$-plonk- $B$. The extension is easily shown to be conservative, and we may, therefore, say "There is a connective having these properties". But is there only one? It seems rather odd to say we have defined plonk unless we can show that $A$-plonk- $B$ is a function of $A$ and $B$, i.e., given $A$ and $B$ there is only one proposition $A$-plonk- $B$. But what do we mean by uniqueness when operating from a synthetic, contextualist point of view? Clearly that at most one inferential role is permitted by the characterization of plonk; i.e., that there cannot be two connectives which share the characterization given to plonk but which otherwise sometimes play different roles. Formally put, uniqueness means that if exactly the same properties are ascribed to some other connective, say plink, then $A$-plink- $B$ will play exactly the same role in inference as $A$-plonk- $B$ both as premiss and as conclusion. [3, page 133]

The idea here is straightforward. Inference rules only truly define a connective if they go beyond describing some of its inferential properties, and go on to determine its behaviour in valid argument. This is thecriterion of UNIQUENESS. Keeping on using Belnap's mathematical analogy, we will call his first criterion the EXISTENCE criterion. The antecedently given context of deducibility provides the space for the definition of some concept (it allows for its existence), if it may be conservatively extended to include it. The concept is defined uniquely if whenever we attempt to define it $t$ wice, the two concepts defined agree.

In the rest of this paper, I will use Belnap's criteria of existence and uniqueness to examine four different issues in the philosophy of logic, concerning a connective $(A)$, a quantifier $(\forall)$, a variable binding term operator $(\varepsilon)$ and a modal operator $(\square)$. Along the way we will learn more about connectives, quantifiers, variable binders and modal operators, and we will also hopefully learn more about what is involved in using inference rules to define.

§1. $\wedge$. If you were forced to pick an unproblematic binary connective, conjunction would be a smart choice. For the conditional, there is no critical consensus: some defend of the material conditional, others propose some kind of modal or intensional analysis, and yet others think that the conditional is not really a connective at all, but a complicated speech-act more like conditional assertion than anything else. Even disjunction has its own disputes--how are 
we to mark the difference (if there is a real difference) between inclusive and exclusive disjunction? If I use "or" in an exclusive way, does this imply or merely presuppose that the disjuncts are not both true? There are no disputes like these over conjunction. The rules $[\wedge I]$ and $[\wedge E]$ given on page 204 are not that complicated and are not in that much dispute. However, there is some dispute over the meaning of "and" and Belnap's two criteria give us a way to understand the kinds of discussions concerning it.

Existence. First, consider the existence problem. Do the rules $[\wedge I]$ and $[\wedge E]$ conservatively extend a proof system to which they are added? The answer would seem to be affirmative, since Prawitz has taught us that natural deduction systems for intuitionistic logic or for classical logic, featuring just these rules, have the normalisation property [18]. Any non-normal proof (a proof with adjacent steps where a particle is introduced only to be eliminated straight after) may be converted into a normal proof. This means, in the case of intuitionistic logic and many other logics, that the proof system has the subformula property for normal proofs. If there is a normal proof from premises $X$ to conclusion $A$, then the proof will use only subformulas of the formulas in $X$ or $A$. This means if we have an argument from $X$ to $A$, where $X$ and $A$ do not feature " $\wedge$ ", then a normal proof from $X$ to $A$ will not feature the " $\wedge$ " rules. If for every proof from $X$ to $A$ we can find some normal proof, this tells us that adding the rules for " $\wedge$ " do not let us prove any inferences in the old vocabulary that we couldn't prove before. Conjunction is a conservative extension. It passes the existence test. Or does it?

Results in proof theory like these are notoriously sensitive to being formulated precisely. Normalisation results are no exception. As a matter of fact, the rules $[\wedge I]$ and $[\wedge E]$ do not conservatively extend all proof systems. Consider a very basic proof system, in the style of Gentzen [11, 12] or Prawitz [18]. Proofs are trees of formulas, with premises as leaves and conclusion as the root. The identity proof for a formula $A$ is the simple tree

whose root is identical to its only leaf-the formula $A$ itself. The rules for conjunction are as given on page 204 . We can, for example, construct a proof whose premises are instances of $p \wedge(q \wedge r)$ and whose conclusion is $(p \wedge q) \wedge r$ by composing many $[\wedge I]$ and $[\wedge E]$ steps

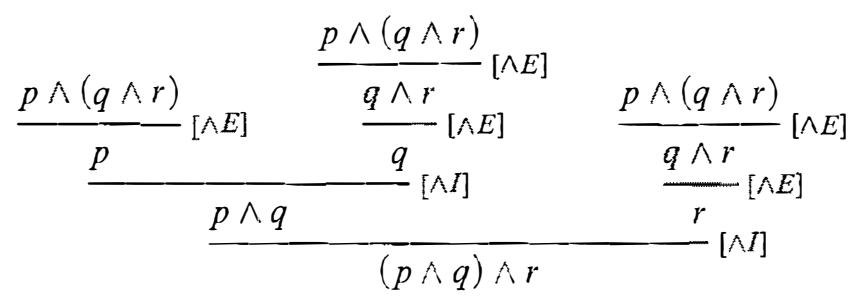


The result is a proof system for lattice conjunction. However, in this little system, the primitive system (with identity as its only rule) is not conservatively extended by the conjunction rule, at least when you look at matters closely. The reasoning is straightforward. In the system with conjunction rules, we have a proof whose premises are $p$ and $q$ and whose conclusion is $q$.

$$
\frac{p \quad q}{\frac{p \wedge q}{q}[\wedge I]}[\wedge E]
$$

There is no such proof that avoids using the conjunction rules. In this system, the only proof ending in $q$ that uses no connectives is the identity proof in which $q$ is the sole premise and conclusion.

So, we have seen a way in which even rules such as $[\wedge I]$ and $[\wedge E]$ may fail Belnap's existence test. The case may seem ad hoc, but it is not as cooked up as it seems. If, for example, you have concerns for relevance [1, 2, 8, 21] in a consequence relation, then you may think that you do not have a proof from $p$ together with $q$ to the conclusion $q$. There may be a proof from $q$ to $q$ (the identity proof we have already mentioned), but bringing the extra premise $p$ alongside the other premise does not necessarily involve it in the proof. If our antecedently given context of deducibility respects this strict criterion of relevance in this way, then the conjunction rules $[\wedge I]$ and $[\wedge E]$ are to be rejected as definitions - they fail Belnap's existence criterion ${ }^{2}$.

Uniqueness. We may say something about uniqueness as an illustration of the other criterion. If we are happy with $[\wedge I]$ and $[\wedge E]$ as rules, and our system admits them because we were already committed to the validity of the argument from $p, q$ to $p$, then we may ask if the rules merely describe conjunction or truly define it. So suppose that we introduce two conjunction connectives $\wedge$ and $\&$, with the same form of rules. Are they equivalent?

We have the following proofs:
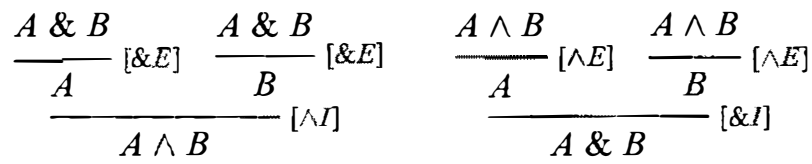

So $\wedge$ is interchangeable with $\&$ as a premise or a conclusion in any argument. They are equivalent. If we have a point at which we require $A \wedge B$ as a premise, we may justify it on the basis of the assumption of $A \& B$, and vice versa.

\footnotetext{
${ }^{2}$ This happens in real-life natural deduction systems. In Lemmon's Beginning Logic [16], the only way to derive the classically valid inference from $p$ to $q \supset p$ is via a conjunction such as $p \wedge q$. There is no way to vacuously discharge a hypothesis $q$ that is not actually present as a premise, and the only way to involve it as a premise is to use another connective such as conjunction. So, either Lemmon's rules for $\supset$ are incomplete, or, in his system, the conjunction rules fail Belnap's test.
} 
Similarly, if we wish to derive $A \wedge B$, we may derive $A \& B$ and deduce $A \wedge B$ from it, using these proofs ${ }^{3}$.

So, we have uniqueness. Does this uniqueness result mean that any two connectives introduced with these rule have the same meaning? To decide this would take us too far away into general considerations in the theory of meaning. All we have seen so far is that $\wedge$ and $\&$ would play the same role in deductive inference. Perhaps this does not mean that they are fully interchangeable in every context in every respect. For example, you might think of $\wedge$ as "and" and \& as "but." Perhaps these have the same deductive power. Perhaps it is always legitimate to infer $p$ butq from the premises $p$ and $q$, even if it is odd to say it in cases where $q$ is not surprising given the assumption of $p$. If this is the case (and some theories of "but" distinguish it from "and" not in what it says but in what uses of it presupposes), then it may well be that "and" and "but" agree when it comes to inferential power and derivability, but they do not agree in every aspect of meaning. If this is the case, then inferential equivalence can define a connective like conjunction up to a point, but it cannot determine its meaning in any sense more precise than that.

$\S 2 . \forall$. Now let us consider the universal quantifier. It has two relatively straightforward inference rules

$$
\frac{A[c]}{(\forall x) A[x]}[\forall I] \quad \frac{(\forall x) A[x]}{A[t]}[\forall E]
$$

These rules are relatively straightforward, but they have complexities beyond the rules of a propositional logic. These rules appeal to the syntax of names and variables. In $[\forall E]$, we may derive $A[t]$ (a sentence in which the singular term $t$ may appear some number of times), from the premise $(\forall x) A[x]$, where $A[x]$ is found by replacing the instances of $t$ in $A[t]$ by the variable $x$. The $[\forall E]$ rule is simple. We infer from a universally quantified claim any of its instances.

The more complicated rule is the introduction rule $[\forall I]$. Here, we don't derive $(\forall x) A[x]$ from $A[c]$ by itself. We may only derive $(\forall x) A[x]$ if we have derived $A[c]$ in a special way. We can make this inference when we have derived $A[c]$ from premises in which the name $c$ does not occur. The idea is straightforward. If we had any other object (call it $d$ ), then we could derive $A[d]$ in just the same way from just the same premises. If we presumed nothing "about $c$," then the proof of $A[c]$ is completely general and could have served

\footnotetext{
${ }^{3}$ There is, however, a little subtlety in doing this. In the Gentzen-Prawitz-style system I am using, we trade in the use of the premise $A \wedge B$ by two appeals to the premise $A \& B$. If our antecedent theory of deducibility distinguishes multiplicities of premises, then $A \& B$ and $A \wedge B$ need not be equivalent. For those keeping score of structural rules appealed to [21], for existence we used WEAKENING, and for uniqueness, we need CONTRACTION.
} 
as well as a proof of $A[d]$, or of $A[x]$ for any object $x$. In other words, this gives us grounds to conclude $(\forall x) A[x]$.

Existence. The standard normalisation argument in natural deduction or cut elimination argument sequent systems show that the addition of these rules in well-behaved proof systems is conservative. Adding these quantifier rules in sensible proof theories is always conservative, we pass Belnap's existence test. The crucial step in a normalisation proof shows us that if we have a proof in which a universal quantifier is introduced only to be eliminated immediately at the next step.

$$
\begin{gathered}
X \\
\vdots \pi \\
\frac{A[c]}{(\forall x) A[x]} \\
\frac{A[d]}{\forall I]}[\forall E]
\end{gathered}
$$

Here, to justify the appeal to $[\forall I]$, we know that $c$ does not appear in the proof $\pi$ in the premises $X$. This means that we may replace $c$ by $d$ in the proof $\pi$ to get

$$
\begin{gathered}
X \\
\vdots \pi_{d}^{c} \\
A[d]
\end{gathered}
$$

which is a proof of the same conclusion $A[d]$ from the same premises $X$, without going through the detour formula $(\forall x) A[x]$. The side condition on $[\forall \mathrm{I}]$ is just what we need to prove existence.

Uniqueness. The curious feature here is uniqueness. Think of the kind of argument that would show that two universal quantifiers - let's say, $(\forall x)$ and $(x)$ - are equivalent. The simplest such argument would go like this:

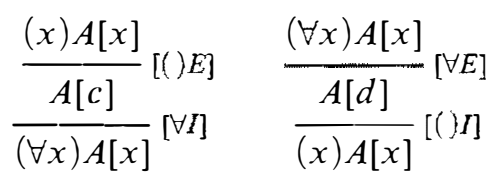

These arguments succeed only under certain conditions. The first proof, from $(x) A$ to $(\forall x) A$, works only if the name $c$ substituted for $x$ in $[() E]$ is also allowed to count as a term appropriate for substitution in the rule $[\forall I]$. The second proof, from $(\forall x) A$ to $(x) A$, works only if the name $d$ substituted for $x$ in $[\forall E]$ is also allowed to count as a term appropriate for substitution in the rule $[() I]$. Only when the terms used in the rules for one quantifier agree with the terms used in the other can we manage to prove equivalence.

This restriction is important. Many sorted predicate logics may have different universal quantifiers corresponding to different domains. Each quantifier 
will satisfy rules like $[\forall I / \forall E]$ without the quantifiers coinciding. The inferences from one quantifier to another would be blocked, because we cannot step from $(\forall x) A[x]$ to $A[c]$ to $(x) A[x]$. The problem is not that the inference steps from truth to falsity at any point - it is that the result is syntactically illformed. To make matters precise, suppose we have a language with two kinds of singular terms (for simplicity, let us say that they are disjoint - no singular term is of both kinds), $a$-terms and $b$-terms, and we correspondingly have $a$ variables and $b$-variables. The syntax of each $n$-place predicate is determined not only by its arity, but also by the kind of each place. There may be 1-place predicates which take $a$-terms and 1 -place predicates which take $b$-terms. Call these $\langle a\rangle$-predicates and $\langle b\rangle$-predicates respectively. Similarly, an 2-place predicate may be an $\langle a, a\rangle$ predicate, an $\langle a, b\rangle$-predicate, a $\langle b, a\rangle$-predicate or a $\langle b, b\rangle$-predicate, and so on. The rules for forming complex formulas are as usual, but we have the following restrictions on quantifiers. If $A[c]$ is a sentence, in which an $a$-term $c$ occurs, then $\left(\forall_{a} x\right) A[x]$ is a sentence, where $A[x]$ is the result of replacing the $a$-term $c$ in $A[c]$ by the $a$-variable $x$. Similarly, if $d$ is a $b$-term, then $\left(\forall_{b} y\right) A[d]$ is a sentence, in which we quantify into $b$-term position. Now the inference from $\left(\forall_{a} x\right) F x$ to $\left(\forall_{b} y\right) F y$ will fail, not because the premise is true and the conclusion is false, but if the premise $\left(\forall_{a} x\right) F x$ is syntactically well-formed, then the conclusion $\left(\forall_{b} y\right) F y$ is not. For $\left(\forall_{a} x\right) F x$ to make sense, $F$ must be an $\langle a\rangle$-predicate. For $\left(\forall_{b} y\right) F y$ to make sense, $F$ must be a $\langle b\rangle$-predicate. In the language under discussion, nothing is both.

So what, then, of Belnap's criteria? Do quantifiers pass the uniqueness test? Does the possibility of having two different universal quantifiers mean that we should banish $\forall$ and $\exists$ from the canon of logical constants? Surely such a conclusion is too extreme. However, we must acknowledge that in the face of considerations like this, the choice of quantifiers as logical constants is relative to the syntax of the language under consideration. Once we identify a category of singular terms (of names or variables or whatever), then relative to this choice, the quantifiers (for that category) are logical constants. Existence and uniqueness proofs work, and the meanings of the quantifiers are fixed.

This means, of course, that the choice of syntax has a burden to carry. In some cases - such as mathematical reasoning — the task does not seem too onerous to discharge. In the language of set theory, for example, it is not too hard to discern what singular terms are in use ${ }^{4}$.

For inferentialists, who wish to use rules like these to determine meanings, the identification of the category of singular terms is a matter of pressing importance. For example, Brandom devotes many pages of Making it Explicit [5]

\footnotetext{
${ }^{4}$ Though it is not without its share of controversy. Are class terms able to be paraphrased away without appeal to singular terms for classes? If so, well and good, ZFC and theories without classes seem sufficient. If not, then perhaps NBG and theories with classes, or more radical views are appropriate $[9,17]$.
} 
to the issue of determining the characteristics of singular terms. Figuring out what these are, and what reason we might have for a single category of themrather than multiple disjoint classes-is a pressing project for those who take first-order quantification to tell us something about ontology [20]. Getting the category right tells us something about the significance of taking $\forall$ as a logical constant and something of the significance of taking it to be defined by its inference rules. This attempt at a definition only succeeds given the analysis of singular terms of the language.

§3. $\varepsilon$. A more controversial concept in the vicinity of first-order predicate logic is Hilbert's " $\varepsilon$ ", the variable-binding term operator of indefinite choice. The syntax is relatively straightforward. If $A[t]$ is a sentence in which term $t$ appears, then $\varepsilon x A[x]$ is a singular term, where $A[x]$ is given by replacing the term $t$ in $A[t]$ by $x$. The intended interpretation is that if there is some $x$ such that $A[x]$, then $\varepsilon x A[x]$ denotes some such object. (If there is no such $x$, then the denotation of $\varepsilon x A[x]$ is not so important.) With this understanding of $\varepsilon$, the universal and existential quantifiers may be defined. We have

$$
\begin{array}{rlr}
(\exists x) A[x] & { }_{\mathrm{df}} & A[\varepsilon x A[x]] \\
(\forall x) A[x] & { }_{\mathrm{df}} & A[\varepsilon x \neg A[x]]
\end{array}
$$

Choice is useful for a number of different reasons. Hilbert's original motivation was to do without the quantifiers. You may also be interested in choice in order to more straightforwardly model natural deduction reasoning-it is quite tempting to be able to infer from $(\exists x) A[x]$ the conclusion $A[t]$ for some term $t$ (many students prefer to make a step like this than to use the Official $[\exists E]$ Rule from their natural deduction system). Choosing $\varepsilon x A[x]$ for $t$ makes this inference valid. From $(\exists x) A[x]$ we may infer $A[\varepsilon x A[x]]^{5}$ Another use for $\varepsilon$ may be found in formulating and defending choice principles in mathematical theories. If the syntax allows us to formulate arbitrary choices, then we have scope for "proving" the axiom of choice in some set theory on the basis of more fundamental axioms or principles [7]. The general strategy is clear: if we need to show that!

$$
(\forall x)(\exists y) F x y \supset(\exists f)(\forall x) F x f(x)
$$

- if $f$ is to be a choice function, where $f(x)$ is a choice of a representative $y$ where $F x y$, then taking $f(x)$ to be $\varepsilon y F x y$ has the desired behaviour, since we may prove

$$
(\forall x)(\exists y) F x y \supset(\forall x) F x(\varepsilon y F x y)
$$

\footnotetext{
${ }^{5}$ However, this matter is subtle [13]. It is especially difficult in the presence of intensional operators such as $\square$ and $\bigcirc$. If $\circlearrowleft(\exists x) A[x]$, we may infer $\bigcirc A[\varepsilon x A[x]]$. It looks to all the world that we may infer that there is an object $c$ (the denotation of the term $\varepsilon x A[x]$ in $\supset A[\varepsilon x A[x]]$ ) such that $\bigcirc A[c]$, in other words, $(\exists x) \bigcirc A[x]$. But isn't the inference from $\diamond(\exists x) A[x]$ to $(\exists x) \bigcirc A[x]$ invalid?
} 
So, with these thoughts in mind, it is appropriate to ask the question of what kind of concept $\varepsilon$ turns out to be, to better understand what is involved in admitting it into our vocabulary. As with quantification, issues arise with uniqueness more than with existence. There is no doubt that $\varepsilon$ may be conservatively extended to the language of first-order logic - at least if we admit the axiom of choice into our metatheory. Any model of the language of first order logic may be extended with a choice function selecting from the extension of a formula $A[x]$ with one free variable, an object to be the denotation of $\varepsilon x A[x]$. This kind of model not only validates the conditions on $\varepsilon$ we have already seen, but also stronger conditions such as "left uniqueness"

$$
(\forall x)(A[x] \equiv B[x]) \supset \varepsilon x A[x]=\varepsilon x B[x]
$$

to the effect that if $A$ and $B$ are coextensive predicates, then the choice for $A$ is identical to the choice for $B^{6}$. So, adding choice, with quite strong principles, is conservative over a base logic like classical predicate logic. Any troubles with $\varepsilon$ do not arise over questions of existence. Uniqueness is another matter.

Uniqueness. Suppose that we add two $\varepsilon$ operators to the language. We do not need to fix on a particular collection of inference rules for $\varepsilon$, but a plausible pair of rules for $\varepsilon$ are these:

$$
\frac{A[c]}{A[\varepsilon \times A[x]]}[\varepsilon I] \quad \frac{[A[c]]}{\vdots \pi} \quad \frac{A[\varepsilon \times A[x]]}{C} \stackrel{C}{C} \text { (c occurs in no other premise in } \pi \text {.) }
$$

These rules together ensure that $\varepsilon$ makes $\exists x A[x]$ equivalent to $A[\varepsilon x A[x]]$. However, they are not enough to assure uniqueness. They may both be satisfied by wildly different choice functions. For example, if we have a model of predicate logic, we may interpret a choice function by selecting a member of every non-empty subset of the domain. Such choices may be made in very many different ways and, and so, we may have very many different interpretations of the choice function. For example, take a model of the language of arithmetic modulo 3 , with the domain $\{0,1,2\}$, the arithmetic vocabulary of $1,+, \times$ with the interpretation of of arithmetic mod three. Take two choice functions, interpreting the two choice operators $\varepsilon$ and $\varepsilon^{\prime}$. For $\varepsilon x A[x]$, we choose the smallest ${ }^{7} x$ satisfying the property $A[x]$, if there is one, and 0 if there is not. For $\varepsilon^{\prime} x A[x]$, we choose the largest $t^{8} x$ satisfying $A[x]$, if there is one, and 2 if there is not. We can see under this interpretation that the rules $[\varepsilon I]$ and $[\varepsilon E]$ are satisfied for both $\varepsilon$ and $\varepsilon^{\prime}$, and we have left uniqueness too. Are $\varepsilon$ and $\varepsilon^{\prime}$ in any way equivalent? Can we substitute $\varepsilon$ for $\varepsilon^{\prime \prime}$ ?

\footnotetext{
${ }^{6}$ For a recent discussion of left uniqueness and other conditions on $\varepsilon$. see Claus-Peter Wirth's interesting recent paper [24].

${ }^{7}$ Smallest under the ordering $0<1<2$, that is.

${ }^{8}$ Largest under the same ordering: $<1<2$.
} 
No, we cannot. In this interpretation, $\varepsilon x(x=x)$ denotes 0 , and $\varepsilon^{\prime} x(x=x)$ denotes 2 . So, in this interpretation, we have

$$
\varepsilon x(x=x) \neq \varepsilon^{\prime} x(x=x)
$$

but we do have

$$
\varepsilon x(x=x)=\varepsilon x(x=x) \text { and } \varepsilon^{\prime} x(x=x)=\varepsilon^{\prime} x(x=x)
$$

So, $\varepsilon$ is in no way equivalent to $\varepsilon^{\prime}$. If $I$ learn to use $\varepsilon$ by way of inference rules like $[\varepsilon I]$ and $[\varepsilon E]$ (and left uniqueness and perhaps other conditions also satisfied in our models), and you do too, then there is no guaran tee that our use of the concept will agree. I could "mean" $\varepsilon$ and you could "mean" $\varepsilon$ '. Our usage will not converge on the one interpretation. Even given the choices we made to fix the logical vocabulary of singular terms, in order to give uniqueness for the quantifiers, we cannot assume that we have uniqueness for choice. Choice does not pass the uniqueness test, and it cannot be thought to be a logical constant in the same manner as the connectives and the quantifiers, unless we either set aside the criterion of uniqueness (so our inference principles do not need to determine the interpretation of the concept), or we find some other concept upon which to ground the interpretation of $\varepsilon$.

Our last example will be another case in which uniqueness is at risk, and in which we may perhaps find the resources to restore uniqueness.

§4. $\square$. Consider modality: in particular, consider a normal modal operator $\square$ - necessity —interpreted as satisfying the simple modal logic $\mathrm{S} 5[4,14,15]$. As is well known, there are many different models for the modal logic S5. If we think of Kripke models in which formulas are evaluated at "worlds", then given any equivalence relation $\approx$, if we take $\square A$ to hold at world $w$ iff $A$ holds at every world $v \approx w$, then the result is a model of the logic S5. A universal model is one in which the equivalence relation $\approx$ on the domain $W$ of worlds is the total relation: $w \approx v$ always. So, in a universal model, $\square A$ is true at some world if and only if $A$ is true at every world.

Now, it appears that these simple facts show that $\square$ is not a true logical constant in our strict sense. We may interpret $\square_{1}$ and $\square_{2}$, both satisfying the rules of the logic S5, with different equivalence relations $\approx_{1}$ and $\approx_{2}$ on the one model. The logic S5 does not force a unique interpretation on our two operators, and there is no way to force $\square_{1}$ and $\square_{2}$ to be equivalent, from the assumption that they are both S5 necessity operators. To put it starkly, the inference rules for $\square$ according to S5 describe the behaviour of $\square$ but they do not define it.

We could leave matters there and feel satisfied that we have a neat answer, which puts $\wedge$ and $\forall$ on one side of the fence (logical constants, fully defined by their inference rules) and $\varepsilon$ and $\square$ on the other (requiring more to define them that their inferential properties alone). Such a position is consonant with the 
main tradition in contemporary modal logic, where we admit that there are many operators satisfying the rules of this or that modal logic, and there are many ways that different modal operators might interact ${ }^{9}$. However, ending here would obscure one important point. The case of $\square_{1}$ and $\square_{2}$ is analogous to the case of the universal quantifier in a multi-sorted vocabulary. We could only prove uniqueness for a universal quantifier relative to a particular choice of syntax - a choice of singular terms and variables. If the analogy with quantifiers holds, perhaps we can make a similar choice in the case of modality. In this modal case, the choice is not so much in the syntax of formulas themselves (in propositional modal logic we have no terms denoting worlds or any other modal "ontology" or "ideology") it must be elsewhere. One possible place to find such a choice of syntax is found in a proof theory for the modal logic S5 I introduced at Logic Colloquium in 2005 [22]. Here, we extend Gentzen's sequent calculus to deal with hypersequents (multisets of sequents), where just as the sequent

$$
X \vdash Y^{r}
$$

tells us that asserting each sentence in $X$ and denying each sentence in $Y$ is ruled out as inconsistent on logical grounds, in the hypersequent appropriate for modal reasoning considering hypothetical situations, or reasoning with different dialogical contexts,

$$
X_{1} \vdash Y_{1}\left|X_{2} \vdash Y_{2}\right| \cdots \mid X_{n} \vdash Y_{n}
$$

tells us that asserting $X_{1}$ and denying $Y_{1}$ in one context, together with asserting $X_{2}$ and denying $Y_{2}$ in another context $\ldots$ and asserting $X_{n}$ and denying $Y_{n}$ in another is inconsistent on logical grounds. The general principle is best illustrated by a derivation of a principle of $\mathrm{S} 5$, to infer $\square-\square \neg A$ from $A$.

$$
\begin{gathered}
\frac{A \vdash A}{A, \neg A \vdash-}[\neg L] \\
\frac{A \vdash \mid \square \neg A \vdash}{A \vdash \mid \vdash \neg \square \neg A}[\neg R] \\
\frac{A \vdash \mid \vdash \square \neg \square \neg A}{A \vdash \square \neg \square \neg A}[\text { merge }]
\end{gathered}
$$

In the second line we have $A, \neg A \vdash \_$asserting both $A$ and $\neg A$ is ruled out. At the third line, we move to $A \vdash \mid \square \neg A \vdash$, which tells us that if we assert $A$ on one context, and in another assert $\square \neg A$, this is also ruled out. This is what makes $\square$ modal. Taking $\neg A$ to be necessary is inconsistent with supposing (in some other "possibility") that it is the case that $A$. The next line moves

\footnotetext{
${ }^{9}$ The canonical text on mutimodal logics is Gabbay, Kurucz, Wolter and Zakharyashev's Many-Dimensional modal logics [10].
} 
us from the assertion of $\square-\neg A$ to the denial of,$- \square-1 A$, and then after that, if asserting $A$ (in some context) is inconsistent with denying $-\square-\neg A$ (in another context), then it is also inconsistent with denying $\square-1 \square-1 A$, because denying $\square B$ in general has the same import as denying $B$ in some other arbitrary context. Finally, if it is inconsistent to assert $A$ in some context and deny $\square-\neg-\neg A$ in another, it is just as bad to assert $A$ and deny $\square-\neg \square \neg A$ in the very same context, and hence, we have $A \vdash \square-1 \square-\neg A$, the conclusion.

The rules for $\square$ used here take this form:

$$
\frac{X, A \vdash Y \mid \Delta}{\square A \vdash|X \vdash Y| \Delta}[\square L] \quad \frac{\vdash A \mid \Delta}{\vdash \square A \mid \Delta}[\square R]
$$

In the earlier paper I show that this hypersequent calculus admits of a cut elimination proof (and hence, we can show existence), and that it is indeed a system sound and complete for S5. What is more interesting from our point of view is the fact that we can prove uniqueness too.

Uniqueness. If $\square_{1}$ and $\square_{2}$ use the same sequent rules, we may prove equivalence of $\square_{1}$ and $\square_{2}$ as follows:

$$
\frac{\frac{A \vdash A}{\square_{1} A \vdash \mid \vdash A}\left[\square_{1} L\right]}{\frac{\square_{1} A \vdash \mid \vdash \square_{2} A}{\square_{1} A \vdash \square_{2} A}\left[{ }_{2} R\right]}[\text { merge }]
$$$$
\frac{\frac{A \vdash A}{\square_{2} A \vdash \mid \vdash A}\left[\square_{2} L\right]}{\frac{\square_{2} A \vdash \mid \vdash \square_{1} A}{\square_{2} A \vdash \square_{1} A}\left[\square_{1} R\right]}\left[\begin{array}{l}
\text { merge }] \\
\end{array}\right.
$$

This is not a difficult result. The technique is identical to that used when we proved that two universal quantifiers are equivalent. In that case, we relied upon the fact that the quantifiers trade on the one syntactic category of names and variables. In this case, we rely upon the fact that the two modal operators trade upon the one hypersequent structure. In the case of the quantifiers, uniqueness may be defended on the basis of the uniqueness of syntactic category of names and variables in the language under consideration. If the analogy is to be made out, the same thing may be said for modality, except (if the interpretation of the hypersequent proof theory I hastily sketched is accepted) the defence is not made on the basis of any syntactic feature of the sentences of the language in question, but rather, on the way we combine them in dialogue. The modal operator $\square$ gains its interpretation, on this view, on the basis of the dialogical shifts from context to context made in modal reasoning. On this view, any stability we have in our understanding of the concept of necessity and possibility may be found not in the shared access we have to the one domain of possible worlds (how could we have access to that, except via or modal reasoning?) but in the way we govern shifts in dialogue when we together consider what would happen if ...

Belnap's criteria of existence and uniqueness were offered as a way to draw a principled boundary that would keep logical operators on one side of the 
fence, while keeping anomalous definitions like that of tonk on the other side. These criteria are fruitful not only in the case of barring monsters like tonk, but they also have something to teach us in the more prosaic cases of $\wedge, \forall, \varepsilon$ and $\square$.

Acknowledgments. Thanks to Allen Hazen, Lloyd Humberstone, Penelope Maddy, Albert Visser, Alasdair Urquhart, Heinrich Wansing and audiences at the University of Melbourne Logic Group, Logica 2007, Logic Colloquium 2007 for discussion on these topics. This research is supported by the Australian Research Council, through grant DP0343388, and Ethel's self-titled album.

\section{REFERENCES}

[1] Alan Ross Anderson and Nuel D. Belnap, Jr., Entailment: The Logic of Relevance and Necessity, Volume I, Princeton University Press, Princeton, N. J., 1975, With contributions by J. Michael Dunn and Robert K. Meyer, and further contributions by John R. Chidgey, J. Alberto Coffa, Dorothy L. Grover, Bas van Fraassen, Hugues LeBlanc, Storrs McCall, Zane Parks, Garrel Pottinger, Richard Routley, Alasdair Urquhart and Robert G. Wolf.

[2] Alan Røss Anderson, Nuel D. Belnap, Jr., and J. Michael Dunn, Entailment. The Logic of Relevance and Necessity. Volume II, Princeton University Press, Princeton, NJ, 1992, With contributions by Kit Fine, Alasdair Urquhart et al, Includes a bibliography of entailment by Robert G. Wolf.

[3] Nuel D. Belnap, Tonk, Plonk and Plink, Analysis, vol. 22 (1962), pp. 130-134.

[4] Patrick Blackburn, Maarten de Ruje, and Yde Venema, Modal Logic, Cambridge Tracts in Theoretical Computer Science, vol. 53. Cambridge University Press, Cambridge, 2001.

[5] Robert B. Brandom, Making it Explicit, Harvard University Press, Cambridge, Massachusetts, 1994.

[6] - Articulating Reasons: An Introduction to Inferentialism, Harvard University Press, Cambridge, Massachusetts, 2000.

[7] JoHn P. Burgess, E pluribus unum: plural logic and set theory, Philosophia Mathematica. Philosophy of Mathematics, its Learning, and its Application. Series III, vol. 12 (2004), no. 3, pp. 193-221.

[8] J. Michael Dunn and Greg Restall, Relevance logic, Handbook of Philosophical Logic (Dov M. Gabbay, editor), vol. 6, Kluwer Academic Publishers, London, UK, second ed., 2002, pp. 1-136.

[9] T. E. Forster, Set Theory with a Universal Set, second ed., Oxford Logic Guides, vol. 31, The Clarendon Press Oxford University Press, New York, 1995, Exploring an untyped universe, Oxford Science Publications.

[10] D. M. Gabbay, A. Kurucz, F. Wolter, and M. Zakharyaschev, Many-Dimensional Modal Logics: Theory and Applications, Studies in Logic and the Foundations of Mathematics, vol. 148, North-Holland, Amsterdam, 2003.

[11] Gerhard Gentzen, Untersuchungen über das logische Schließen. I, Mathematische Zeitschrift, vol. 39 (1935), no. 1, pp. 176-210.

[12] - - Untersuchungen ïber das logische Schließen. II, Mathematische Zeitschrift, vol.39 (1935), no. 1, pp. 405-431.

[13] Allen HAZEN, Natural deduction and Hilbert's E-operator, Journal of Philosophical Logic, vol. 16 (1987), no. 4, pp. 411-421.

[14] G. E. Hughes and M. J. Cresswell, An Introduction to Modal Logic, Methuen, London, 1968. 
[15] - A New Introduction to Modal Logic, Routledge, London, 1996.

[16] E. J. LEMMON, Beginning Logic, revised ed., Hackett, Indianapolis, 1978, Edited by George W. D. Berry.

[17] Penelope Maddy, Proper classes, The Journal of Symbolic Logic, vol. 48 (1983), no. 1, pp. 113-139.

[18] Dag Prawitz, Natural Deduction. A Proof-Theoretical Study, Acta Universitatis Stockholmiensis. Stockholm Studies in Philosophy, vol. 3, Almqvist and Wiksell, Stockholm, 1965. 39.

[19] Arthur N. Prior, The runabout inference-ticket, Analysis, vol. 21 (1960), no. 2, pp. 38

[20] W. V. O. Quine, On What There Is, Review of Metaphysics, vol. 2 (1948), no. 5, pp. 21-38.

[21] Greg Restall, An Introduction to Substructural Logics, Routledge, London, UK, 2000.

[22] — - Proofnets for S5: sequents and circuits for modal logic, Logic Colloquium 2005 (Costas Dimitracopoulos, Ludomir Newelski, and Dag Normann, editors), Lecture Notes in Logic, vol. 28, ASL, Urbana, IL, 2008, pp. 151-172.

[23] J. T. Stevenson, Roundabout the Runabout Inference-Ticket, Analysis, vol. 21 (1961), no. 6 , pp. $124-128$.

[24] CIAUS-PETER WIRTH, Hilbert's epsilon as an operator of indefinite committed choice, Journal of Applied Logic, vol. 6 (2008), no. 3, pp. 287-317.

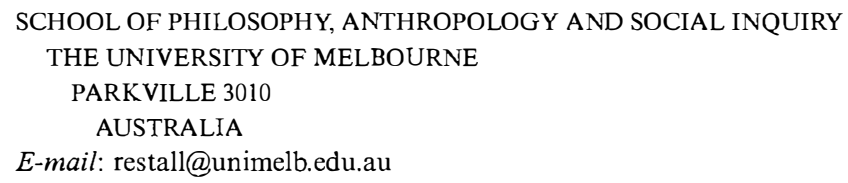




\section{University Library}

\section{- M M I E E R VA A gateway to Melbourne's research publications}

Minerva Access is the Institutional Repository of The University of Melbourne

Author/s:

RESTALL, $G$

Title:

Proof Theory and Meaning: the context of deducibility

Date:

2010

Citation:

RESTALL, G. (2010). Proof Theory and Meaning: the context of deducibility. Logic Colloquium 2007, (1), pp.204-219. Cambridge University Press.

Persistent Link:

http://hdl.handle.net/11343/31741 